1 Universidade de Brasília (UnB) - Brasília (DF), Brasil.

grazifisio3@hotmail.com

2 Universidade de Brasília (UnB) - Brasília (DF)

Brasil.

shimizu@unb.br

3 Universidade de Brasília (UnB) - Brasília (DF),

Brasil.

evertonsilva@unb.br

\section{Atenção Primária à Saúde nos municípios brasileiros: eficiência e disparidades}

\author{
Primary Health Care in Brazilian municipalities: efficiency and \\ disparities
}

Grazielle Custódio David', Helena Eri Shimizu² ${ }^{2}$ Everton Nunes da Silva ${ }^{\mathbf{3}}$

RESUMO O estudo tem por objetivo analisar a eficiência da Atenção Primária à Saúde e de averiguar possíveis disparidades em saúde por meio de um estudo ecológico transversal baseado em dados dos municípios referentes ao IDSUS da atenção primária e no gasto total médio per capita, estratificado por região e grupos homogêneos, para o período 2008-2010. Observouse a dificuldade de estruturação da atenção primária nas grandes cidades e no Norte; o favorecimento das transferências federais aos municípios com maiores necessidades; a maior eficiência foi encontrada no Nordeste e no grupo homogêneo de municípios com os piores indicadores socioeconômicos e de estrutura de saúde. Observaram-se, também, grandes disparidades entre as regiões brasileiras e os grupos homogêneos de municípios, segundo suas características socioeconômicas e de estrutura de saúde, no que se refere à eficiência da Atenção Primária à Saúde e à capacidade de aplicação de recursos próprios pelos municípios. Constatou-se, por fim, a existência de importante inexecução do valor transferido pela União para os municípios dentro do bloco de financiamento da Atenção Básica.

PALAVRAS-CHAVE Atenção Primária à Saúde; Disparidades em assistência à saúde; Economia da saúde.

\begin{abstract}
The study aims to analyze the efficiency of Primary Health Care and to find out possible disparities in health by means of a cross-sectional ecological study based on data from municipalities concerning primary care IDSUS and total expenditure per capita average, stratified by region and homogeneous groups, for the period 2008-2010.It was noted the difficulty in structuring primary care in the municipalities and in the North Region; the favoring of federal transfers to municipalities in greater needs; the greatest efficiencies found in the Northeast Region in the homogeneous group of municipalities with the worst socio-economic and structural health indicators. Large differences were also observed among Brazilian regions and homogeneous groups of municipalities according to their socio-economic characteristics and health structure as regards the efficiency of primary health care and the municipalities' ability to apply their own resources. Finally, it is to be remarked the significant nonuse of the amount transferred by the federal government to the municipalities within the primary care funding block.
\end{abstract}

KEYWORDS Primary Health Care; Healthcare disparities; Health economics. 


\section{Introdução}

Em um contexto em que os recursos são limitados, o Estado deve fazer escolhas ótimas para gerir a oferta de ações e serviços de saúde no intuito de aumentar os resultados à população em termos de longevidade e de qualidade de vida. Nesse sentido, a análise econômica torna-se uma ferramenta importante para definir alternativas eficientes em saúde (BROUSSELLE; LACHAINE; CONTANDRIOPOULOS, 2013).

A eficiência em saúde é entendida como a relação entre o custo e o impacto dos serviços sobre a saúde da população, mantido um nível de qualidade determinado (VIACAVA ET AL., 2012). Eficiência refere-se à capacidade de um sistema de funcionar com menores custos sem sacrificar os resultados, com vistas a obter a melhor relação de custo-efetividade possível (DONABEDIAN, 2003).

São poucos os estudos que avaliam a eficiência em saúde no Brasil. A maioria dos artigos realizados recentemente refere-se à avaliação de ações, procedimentos e serviços de saúde específicos, com média e alta complexidades. Especificamente relacionados à atenção primária, foram encontrados quatro estudos: dois com escopo estadual em São Paulo (VARELA; MARTINS; FÁVERO, 2012) e Espírito Santo (ALVES; COSTA, 2013), um voltado aos municípios da região Sudeste (SANTOS; GONÇALVEZ; FIGUEIREDO, 2013) e o último, aos municípios brasileiros (DIAS, 2010); todos utilizaram como método de avaliação a Análise Envoltória de Dados (DEA).

Os principais achados foram: tanto nos estudos sobre dos municípios paulistas como capixabas, observou-se que somente maior aporte de recursos financeiros não implica maior eficiência; o estudo da região sudeste teve como uma de suas principais contribuições a proposta do modelo analítico de desempenho da alocação de recursos na Atenção Básica da saúde pública; por fim, o estudo que avaliou 4.007 municípios brasileiros observou maior eficiência da Atenção Básica nos municípios da região Nordeste e Norte, dos municípios com melhor saneamento e maior proporção de crianças, enquanto municípios com maior proporção de idosos, capitais de estados, e maior relação de médicos por enfermeiros estavam associados com menor eficiência.

Considerando o aumento do gasto público na área social nas últimas décadas (BRASIL, 2012B), torna-se premente conduzir pesquisas que o avaliem, no intuito de orientar a aplicação mais eficiente dos recursos disponíveis, especialmente os gastos relacionados à atenção primária, que representa a principal estratégia de reorganização do sistema de saúde e cujo propósito é desenvolver atenção integral que impacte na situação de saúde e nos determinantes e condicionantes de saúde das coletividades, de acordo com a Portaria GM/MS n. 2.488/2011.

O financiamento da atenção primária é tripartite, financiado pelos três entes federados, sendo que a União transfere aos municípios os recursos financeiros por meio do bloco de financiamento da atenção primária, na modalidade fundo a fundo, que é constituído pelos pisos da Atenção Básica: i) fixo (PAB fixo), composto por recurso per capita; e ii) variável (PAB variável), composto por recursos destinados à implantação de estratégias prioritárias (PIOLA ET AL., 2009). Os estados também transferem, fundo-a-fundo, recursos financeiros aos municípios por meio do bloco de financiamento da Atenção Básica. Por fim, o município aplica recursos próprios para financiar a Atenção Primária à Saúde (APS).

A expansão da atenção primária tem sido significativa. No final de 2013 , havia 36 mil equipes de Estratégia Saúde da Família (ESF), 300 mil agentes comunitários de saúde e 23 mil equipes de saúde bucal (RODRIGUeS ET AL., 2014). Apesar desses avanços, o seu financiamento tem sido problemático, sobretudo porque ainda é insuficiente (MENDES; MARQUES, 2014).

Apesar das estratégias para aprimorar o financiamento da atenção primária, sabe-se que os municípios convivem com diversos tipos 
de dificuldades, especialmente os de pequeno porte, porque não contam com recursos próprios suficientes para financiar as despesas não cobertas pelo governo federal (MENDES; MARQUES, 2014), o que demanda a necessidade de analisar com maior profundidade uma distribuição mais equitativa dos recursos para o melhor desempenho do sistema de saúde.

Dessa forma, este estudo tem por objetivo avaliar a eficiência econômica da APS nos municípios brasileiros, além de averiguar possíveis disparidades regionais e de fatores socioeconômicos e de características do sistema de saúde, com base no gasto e o desempenho da atenção primária no Brasil no período 2008-2010.

\section{Método}

Trata-se de um estudo ecológico transversal baseado em dados dos municípios brasileiros no período 2008-2010 referentes ao desempenho e aos gastos em saúde, ambos atinentes às ações e serviços da atenção primária. O índice de desempenho da atenção primária e seu respectivo gasto total médio per capita foram estratificados em duas categorias - alto (A) e baixo (B) -, levando-se em consideração a média nacional do período sob investigação. Assim, obtiveram-se quatro grupos:

i) $\mathrm{AA}$, municípios com alto desempenho e alto gasto total médio per capita;

ii) $\mathrm{AB}$, municípios com alto desempenho e baixo gasto total médio per capita;

iii) BA, municípios com baixo desempenho e alto gasto total médio per capita;

iv) $\mathrm{BB}$, municípios com baixo desempenho e baixo gasto total médio per capita.

Para fins desta análise, consideraram-se os municípios do grupo $A B$ eficientes em termos econômicos, visto que obtiveram desempenho acima da média nacional (output) com recursos financeiros per capita aplicados na atenção primária abaixo da média nacional (input); os municípios do grupo BA, por sua vez, foram considerados ineficientes, pois apresentaram desempenho abaixo da média nacional e utilizaram recursos financeiros per capita acima da média nacional.

O Índice de Desempenho do Sistema Único de Saúde da Atenção Primária (IDSUS-AP) foi utilizado como medida de desempenho dos municípios brasileiros e disponibilizado pelo Ministério da Saúde em 2012. É composto por indicadores de acesso e efetividade da atenção primária, a partir dos dados do período de 2007-2010 (BRASIL, 2012A). Esse índice pode assumir valores entre zero (pior desempenho) e dez (melhor desempenho). São dez indicadores que caracterizam o IDSUS-AP, sendo três referentes a acesso: cobertura populacional estimada pelas equipes de atenção primária; cobertura populacional estimada pelas equipes de saúde bucal; e proporção de nascidos vivos de mães com no mínimo sete consultas pré-natal. Os outros sete se referem à efetividade: proporção de internação sensível à atenção primária; taxa de incidência de sífilis congênita; proporção de cura de novos casos de tuberculose pulmonar bacilífera; proporção de cura de novos casos de hanseníase; cobertura com vacina tetravalente em menores de um ano; média da ação de escovação dental supervisionada; e proporção de exodontia em relação aos procedimentos odontológicos. Todos os indicadores tinham meta previamente estabelecida pelo Ministério da Saúde. Assim, resultados que superaram a meta ultrapassaram o índice de $100 \%$.

O gasto total médio per capita foi obtido do Sistema de Orçamentos Públicos em Saúde (Siops) do Ministério da Saúde por meio do bloco de financiamento da Atenção Básica. Optou-se pela média dos valores declarados pelos municípios de 2008 a 2010, de 
forma a manter a correspondência temporal com o IDSUS-AP; o ano de 2007 foi excluído da análise porque 79,3\% dos municípios não declaram o valor despendido na atenção primária, o que enviesaria substancialmente a média amostral dessa variável. Já para os anos 2008, 2009 e 2010, a porcentagem dos municípios que não declaram o valor despendido com APS caiu para 8,8\%, 7,5\% e 6,2\%, respectivamente. Os municípios sem esses dados foram excluídos da amostra; assim, dos 5.565 municípios brasileiros, a amostra do estudo conta com 4.706. O gasto refere-se ao valor total aplicado na atenção primária pelos municípios e é constituído por financiamento tripartite (recursos próprios dos municípios, acrescidos das transferências da União e dos estados). Esses dados foram deflacionados a preços de julho de 2014, utilizando-se a série histórica do Índice Nacional de Preços ao Consumidor Amplo (IPCA) calculado pelo Instituto Brasileiro de Geografia e Estatística (IBGE, 2014).

$\mathrm{O}$ valor transferido pela União para os municípios foi obtido do Siops do Ministério da Saúde por meio do bloco de financiamento da Atenção Básica. A avaliação da execução desse recurso foi realizada pela subtração do valor transferido pela União do gasto total com APS pelo município. Quando o valor do gasto total era inferior ao da transferência, considerou-se como inexecução. É possível que a inexecução das transferências federais seja ainda maior do que o que foi encontrado neste estudo, o que só seria possível avaliar por meio da análise dos fundos municipais de saúde, cujos dados, em muitos casos, ainda não estão disponíveis online.

Para identificar eventuais disparidades regionais, de fatores socioeconômicos e de características do sistema de saúde dos municípios, estes foram agrupados por regiões brasileiras e por grupos homogêneos. Utilizando a metodologia elaborada pelo Ministério da Saúde, este estudo adota a proposta do IDSUS para conformação de grupos homogêneos de municípios. Três índices foram utilizados para caracterizar os agrupamentos: Índice de Desenvolvimento Socioeconômico (IDSE); Índice de Condição de Saúde (ICS) e Índice de Estrutura do Sistema de Saúde do Município (IESSM). Com base nesses índices, criaram-se seis Grupos Homogêneos (GH) (BRASIL, 2012A).

i) GH 1, com 29 municípios, apresenta alto índice de desenvolvimento econômico, médio índice de condição de saúde e alto índice de estrutura do sistema de saúde do município;

ii) GH 2, com 94 municípios, apresenta alto índice de desenvolvimento econômico, médio índice de condição de saúde e médio índice de estrutura do sistema de saúde do município;

iii) GH 3, com 632 municípios, apresenta médio índice de desenvolvimento econômico, médio índice de condição de saúde e baixo índice de estrutura do sistema de saúde do município;

iv) $\mathrm{GH}$ 4, com 587 municípios, apresenta baixo índice de desenvolvimento econômico, baixo índice de condição de saúde e baixo índice de estrutura do sistema de saúde do município;

v) $\mathrm{GH}$ 5, com 2.039 municípios, apresenta médio índice de desenvolvimento econômico, médio índice de condição de saúde e baixíssimo índice de estrutura do sistema de saúde do município, por não apresentar nenhuma estrutura de média e alta complexidade;

vi) GH 6, com 2.184 municípios, apresenta baixo índice de desenvolvimento econômico, baixo índice de condição de saúde e baixíssimo índice de estrutura do sistema de saúde do município, por não apresentar nenhuma estrutura de média e alta complexidade. 
Por se tratar de dados secundários e disponíveis ao público no sítio eletrônico do Ministério da Saúde, não foi necessário submeter este estudo ao Comitê de Ética em Pesquisa.

\section{Resultados}

A tabela 1 apresenta os dados descritivos referentes ao índice de desempenho da atenção primária nos 4.706 municípios brasileiros, os quais refletem informações coletadas no período 2007-2010, além de alguns indicadores individuais sobre acesso e efetividade que compuseram o IDSUS-AP. De um modo geral, a média nacional do IDSUS-AP foi relativamente alta, correspondendo a 8,11 (DP95\% 1,53). Observou-se que os grupos de municípios avaliados com os menores índices de desenvolvimento econômico, de condição de saúde e de estrutura do sistema de saúde foram os que apresentaram maiores notas do IDSUS-AP: grupos homogêneos 5 e 6 obtiveram nota 8,54 (DP95\% 1,34) e 8,25 (DP95\% 1,34), respectivamente; enquanto os grupos homogêneos 1 e 2 obtiveram nota 6,21 (DP95\% 1,52) e 6,7 (DP95\% 1,47), respectivamente. Em termos regionais, o Norte se distancia das demais regiões em relação ao IDSUS-AP, com nota 7,23 (DP95\% 1,91), enquanto as outras ficaram muito próximas à média nacional.
Ao se analisarem os grupos homogêneos referentes aos indicadores selecionados de acesso e efetividade da atenção primária, percebem-se diferenças substanciais. Os grupos homogêneos 5 e 6 ultrapassaram a meta previamente estabelecida de cobertura populacional da atenção primária, com $125,40 \%$ e $115,80 \%$, respectivamente - os valores acima de $100 \%$ ocorrem porque existem mais equipes de saúde da Atenção Básica em relação à população coberta, do que é preconizado pela norma, ao passo que os grupos homogêneos 1 e 2 ficaram abaixo da meta ( $61 \%$ e $64 \%$, respectivamente).

A meta estabelecida para cobertura populacional da saúde bucal era de $50 \%$, a qual foi ultrapassada pelos grupos homogêneos 4, 5 e $6(61,9 \%, 93,5 \%$ e $93,2 \%$, respectivamente). Com relação ao indicador de internação sensível à Atenção Básica, a meta não deveria superar $28 \%$; todavia, somente os grupos homogêneos 1 e 2alcançaram $26,9 \%$ e 28,2\%, respectivamente. A taxa de sífilis congênita é alta em todos os grupos em relação à meta estabelecida de 1 caso em 1.000 nascidos vivos por ano, tendo o grupo homogêneo 3 a menor taxa (1,32/1.000 nascidos) e o grupo homogêneo 1 a maior (2,96/1.000 nascidos). Em relação à cobertura da vacina tetravalente em menores de um ano, apenas o grupo homogêneo 1 não atingiu a meta estabelecida de $95 \%$, apesar de ter apresentado um elevado percentual de cobertura (93\%). 
Tabela 1. Média e desvio padrão dos indicadores selecionados e notas atribuídas aos municípios brasileiros referentes ao Índice de Desempenho do SUS na Atenção Primária (IDSUS-AP), por região brasileira e grupo homogêneo, Brasil, 2007 a 2010

\begin{tabular}{|c|c|c|c|c|c|c|}
\hline \multicolumn{3}{|c|}{ AP - Acesso } & \multicolumn{3}{|c|}{ AP - Efetividade } & IDSUS-AP \\
\hline & (a) & (b) & (c) & (d) & (e) & $(f)$ \\
\hline & $\begin{array}{r}\text { Cob. AP } \\
(\%)\end{array}$ & $\begin{array}{r}\text { Cob. Saúde Bucal } \\
(\%)\end{array}$ & $\begin{array}{r}\text { Tx intern. sens. à } \\
\operatorname{AP}(\%)\end{array}$ & Tx sífilis cong. & $\begin{array}{r}\text { Cob. vacina tetra } \\
(\%)\end{array}$ & IDSUS-AP \\
\hline $\mathrm{n}$ & 4.706 & 4.706 & 4.706 & 1.531 & 4.706 & 4.706 \\
\hline \multicolumn{7}{|c|}{ Média Nacional } \\
\hline & 110,3 & & & 2,20 & & 8,11 \\
\hline Brasil & $(48,43)$ & $83,6(49,15)$ & $37,7(11,20)$ & $(1,40)$ & $107,1(27,12)$ & $(1,53)$ \\
\hline \multicolumn{7}{|c|}{ Grupo Homogêneo } \\
\hline \multirow{3}{*}{1} & 61,0 & & 26,9 & 2,96 & 93,0 & 6,21 \\
\hline & $(20,43)$ & $28,3(16,59)$ & $(5,53)$ & $(2,66)$ & $(5,37)$ & $(1,52)$ \\
\hline & 64,0 & & 28,2 & 1,92 & 95,0 & 6,7 \\
\hline \multirow[t]{2}{*}{2} & $(18,80)$ & $35,3(19,22)$ & $(6,37)$ & $(1,85)$ & $(7,56)$ & $(1,47)$ \\
\hline & 75,3 & & 32,3 & 1,32 & & 7,22 \\
\hline \multirow[t]{2}{*}{3} & $(29,95)$ & $48,3(30,21)$ & $(8,71)$ & $(1,06)$ & $101,0(33,06)$ & $(1,71)$ \\
\hline & 85,0 & & & 1,95 & & 7,34 \\
\hline \multirow[t]{2}{*}{4} & $(31,95)$ & $61,9(32,87)$ & $44,0(11,22)$ & $(1,87)$ & $103,3(23,78)$ & $(1,72)$ \\
\hline & 125,4 & & 34,2 & 2,46 & & 8,54 \\
\hline \multirow[t]{2}{*}{5} & $(56,38)$ & $93,5(55,26)$ & $(9,59)$ & $(0,44)$ & $109,4(25,68)$ & $(1,34)$ \\
\hline & 115,8 & & & 2,81 & & 8,25 \\
\hline \multirow[t]{2}{*}{6} & $(39,54)$ & $93,2(43,84)$ & $41,5(11,22)$ & $(0,96)$ & $108,4(27,54)$ & $(1,34)$ \\
\hline & \multicolumn{6}{|c|}{ Região Brasileira } \\
\hline \multirow{3}{*}{$\mathrm{N}$} & 87,3 & & & 2,21 & & 7,23 \\
\hline & $(38,91)$ & $65,2(40,64)$ & $41,9(11,17)$ & $(1,59)$ & $104,6(26,99)$ & $(1,91)$ \\
\hline & 106,6 & & & 2,38 & & 8,15 \\
\hline \multirow[t]{2}{*}{ NE } & $(32,99)$ & $85,6(39,79)$ & $43,4(11,60)$ & $(1,31)$ & $104,7(23,03)$ & $(1,30)$ \\
\hline & 104,4 & & 37,4 & 2,66 & & 8,28 \\
\hline \multirow[t]{2}{*}{$\mathrm{CO}$} & $(43,83)$ & $87,6(43,66)$ & $(9,73)$ & $(2,30)$ & $109,6(26,18)$ & $(1,22)$ \\
\hline & 116,1 & & 34,0 & 1,64 & & 8,20 \\
\hline \multirow[t]{2}{*}{ SE } & $(59,57)$ & $83,2(56,52)$ & $(9,54)$ & $(1,10)$ & $110,8(32,37)$ & $(1,56)$ \\
\hline & 117,3 & & 33,4 & 2,06 & & 8,35 \\
\hline $\mathrm{S}$ & $(50,31)$ & $84,4(51,86)$ & $(9,46)$ & $(1,05)$ & $104,4(23,52)$ & $(1,46)$ \\
\hline
\end{tabular}

Fonte: Elaboração própria.

Notas: Desvio padrão entre parênteses; (a) cobertura populacional com atenção primária; (b) cobertura populacional com saúde bucal; (c) taxa de internações sensíveis à atenção primária; (d) taxa de sífilis congênita por mil nascidos vivos; (e) cobertura com vacina tetravalente em menores de um ano; ( $f$ ) nota do índice de desempenho do SUS da atenção primária; ( $n$ ) número de observações municipais constantes no IDSUS.

As regiões Sul e Sudeste apresentaram a maior cobertura populacional da atenção primária (117,4\% e $116,1 \%$, respectivamente), enquanto o Norte apresentou a menor $(87,3 \%)$, sendo a única região que ficou abaixo da meta. As regiões Nordeste e Centro-Oeste apresentaram a maior cobertura populacional com saúde bucal ( $85,6 \%$ e $87,6 \%$, respectivamente), enquanto a Norte apresentou a menor $(65,2 \%)$; apesar disso, todas as regiões ultrapassaram a meta de $50 \%$ de cobertura de saúde bucal. $\mathrm{O}$ indicador de internação sensível à atenção primária é mais alto no Nordeste e no Norte (43,4\% e
$41,9 \%$, respectivamente) e mais baixo no Sul e Sudeste (33,4\% e $34 \%$, respectivamente), sendo importante ressaltar que nenhuma das regiões alcançou a meta de ter no máximo $28 \%$ de taxa de internações de condições sensíveis à atenção primária. A taxa de incidência de sífilis congênita é alta em todas as regiões, sendo a menor taxa no Sudeste (1,64 casos/1.000 nascidos) e a maior no Centro-Oeste (2,66 casos/1.000 nascidos). A cobertura da vacina tetravalente está acima da meta em todas as regiões.

A tabela 2 sumaria os dados financeiros médios per capita aplicados na atenção 
primária referentes aos 4.706 municípios brasileiros no período 2008-2010 em relação às transferências da União e ao valor total aplicado nesse nível de densidade tecnológica de atenção à saúde. Em termos nacionais, a média per capita das transferências federais aos municípios foi de R\$ 54,61 (DP95\% 29,71) entre 2008 e 2010, enquanto o valor total médio per capita aplicado na atenção primária foi de $R \$ 136,99$ (DP95\% 164,03). Observouse que as transferências federais seguem um padrão voltado à equidade, pois houve maior repasse de recursos aos municípios com maiores dificuldades no período 20082010, tanto por grupo homogêneo - GH1 $\mathrm{R} \$ 27,38$ (DP95\% 9,61) versus GH6 R\$ 67,07 (DP95\% 27,67) - como por região - Sudeste $\mathrm{R} \$ 46,65$ (DP95\% 168,67) versus Norte R\$ 66,37 (DP 95\% 31,99).

Tabela 2. Média e desvio padrão das transferências federais per capita e do gasto total per capita dos municípios para a atenção primária, por grupo homogêneo e por regiões, Brasil, 2008 a 2010

\begin{tabular}{|c|c|c|c|c|c|c|c|c|}
\hline & \multicolumn{2}{|c|}{2008} & \multicolumn{2}{|c|}{2009} & \multicolumn{2}{|c|}{2010} & \multicolumn{2}{|c|}{$2008-2010$} \\
\hline & (a) & (b) & (c) & (d) & (e) & (f) & (g) & (h) \\
\hline & Transf. fed. & Gasto total & Transf. fed. & Gasto total & Transf. fed. & Gasto total & Transf. fed. média & Gasto total médio \\
\hline & per capita & per capita & per capita & per capita & per capita & per capita & per capita & per capita \\
\hline & $(R \$)$ & $(R \$)$ & $(R \$)$ & $(R \$)$ & $(R \$)$ & $(R \$)$ & $(R \not)$ & \\
\hline \multicolumn{9}{|c|}{ Média Nacional } \\
\hline Brasil & 46,37 & 127,15 & $54,76(30,61)$ & 130,67 & $62,70(33,11)$ & 153,15 & $54,61(29,71)$ & $136,99(164,03)$ \\
\hline & $(28,63)$ & $(309,71)$ & & $(156,25)$ & & $(172,41)$ & & \\
\hline \multicolumn{9}{|c|}{ Grupos Homogêneos } \\
\hline \multirow[t]{2}{*}{1} & $21,21(8,66)$ & $56,58(71,14)$ & $32,93(10,07)$ & 60,45 & 28,00 & 98,56 & 27,38 & $71,86(77,21)$ \\
\hline & & & & $(81,05)$ & $(10,59)$ & $(121,64)$ & $(9,61)$ & \\
\hline 2 & $31,97(10,07)$ & $55,22(71,00)$ & $27,50(11,11)$ & 69,90 & $30,99(11,64)$ & 80,24 & $30,15(10,54)$ & $68,45(67,84)$ \\
\hline & & & & $(84,29)$ & & $(82,42)$ & & \\
\hline \multirow[t]{2}{*}{3} & $27,61(16,19)$ & 80,94 & 33,04 & 94,44 & $37,66(10,50)$ & 114,55 & $32,77(17,56)$ & $96,64(96,56)$ \\
\hline & & $(102,46)$ & $(18,09)$ & $(109,79)$ & & $(129,98)$ & & \\
\hline \multirow[t]{2}{*}{4} & 43,59 & 63,24 & $51,39(21,64)$ & 79,29 & 59,75 & $95,37(76,21)$ & $51,57(21,52)$ & $79,30(56,97)$ \\
\hline & $(20,96)$ & $(77,46)$ & & $(68,25)$ & $(24,30)$ & & & \\
\hline \multirow[t]{2}{*}{5} & 42,82 & 147,83 & 50,20 & 170,65 & 56,55 & 200,25 & $49,85(26,58)$ & $172,91(176,83)$ \\
\hline & $(25,55)$ & $(192,45)$ & $(27,69)$ & $(199,75)$ & $(28,42)$ & $(219,93)$ & & \\
\hline \multirow[t]{2}{*}{6} & 56,53 & 141,97 & $67,10(28,90)$ & 120,70 & $77,58(31,61)$ & 139,28 & $67,07(27,67)$ & $133,98(175,42)$ \\
\hline & $(28,52)$ & $(447,17)$ & & $(121,67)$ & & $(133,07)$ & & \\
\hline \multicolumn{9}{|c|}{ Regiões Brasileiras } \\
\hline $\mathrm{N}$ & 56,69 & $88,69(95,18)$ & 66,10 & 97,02 & $76,34(37,71)$ & 114,16 & $66,37(31,99)$ & $96,88(77,33)$ \\
\hline & $(32,30)$ & & $(32,04)$ & $(89,62)$ & & $(106,71)$ & & \\
\hline NE & 54,76 & 127,52 & 64,83 & 99,62 & $74,60(27,93)$ & 116,21 & $64,68(25,51)$ & $114,00(176,64)$ \\
\hline & $(26,42)$ & $(480,25)$ & $(26,41)$ & $(96,22)$ & & $(104,24)$ & & \\
\hline $\mathrm{CO}$ & 53,06 & 140,61 & $59,75(32,65)$ & 157,65 & 69,84 & 182,81 & $60,88(31,92)$ & $160,35(168,46)$ \\
\hline & $(32,40)$ & $(186,73)$ & & $(174,26)$ & $(33,37)$ & $(190,63)$ & & \\
\hline SE & 39,17 & 132,82 & $46,91(31,31)$ & 154,64 & $53,88(31,28)$ & 182,42 & $46,65(168,53)$ & $156,62(168,67)$ \\
\hline & $(24,59)$ & $(177,68)$ & & $(187,45)$ & & $(59,49)$ & & \\
\hline S & 42,15 & 142,00 & $49,31(25,94)$ & 159,51 & $54,41(26,69)$ & 184,80 & $48,62(24,27)$ & $162,43(147,74)$ \\
\hline & $(23,87)$ & $(161,46)$ & & $(171,68)$ & & $(183,98)$ & & \\
\hline
\end{tabular}

Fonte: Elaboração própria.

Notas: Valores deflacionados a preços de julho de 2014, com base no IPCA-IBGE, seguido do desvio padrão entre parênteses: (a) transferências federais per capita aos municípios brasileiros referentes à atenção primária em 2008; (b) gasto total per capita aplicado na atenção primária pelos municípios brasileiros em 2008 , considerando o financiamento tripartite (União, estado e município), avaliado por meio do bloco de financiamento da Atenção Básica; (c) transferências federais per capita aos municípios brasileiros referentes à atenção primária em 2009; (d) gasto total per capita aplicado na atenção primária pelos municípios brasileiros em 2009 , considerando o financiamento tripartite (União, estado e município); (e) transferências federais per capita aos municípios brasileiros referentes à atenção primária em 2010; (f) gasto total per capita aplicado na atenção primária pelos municípios brasileiros em 2010, considerando o financiamento tripartite (União, estado e município); (g) transferências federais médias per capita aos municípios brasileiros referentes à atenção primária entre 2008 e 2010; (h) gasto total médio per capita aplicado na atenção primária pelos municípios brasileiros entre 2008 e 2010, considerando o financiamento tripartite (União, estado e município). 
Em termos de grupos homogêneos e do gasto total médio per capita com a atenção primária no período 2008-2010, verifica-se que os grupos 5 e 6, mais pobres e com serviços de saúde menos densos tecnologicamente, apresentaram maior valor per capita liquidado, correspondendo a $\mathrm{R} \$$ 172,91 (DP95\% 176,83) e R\$133,98 (DP95\% 175,42), respectivamente; já os grupos homogêneos 1 e 2 , mais ricos e com serviços de saúde mais densos tecnologicamente, liquidaram relativamente menos na atenção primária, com média no período de $\mathrm{R} \$$ 71,86 (DP95\% 77,21) e R $\$ 68,45$ (DP05\% 67,84 ), respectivamente (tabela 2).

Quanto às regiões brasileiras, observa-se dificuldade na contrapartida do gasto total médio per capita com atenção primária pelos municípios das regiões Norte e Nordeste, visto que, apesar de receberem as maiores transferências federais per capita, têm o menor gasto total per capita: Norte $\mathrm{R} \$ 96,88$ (DP95\% 77,03) e Nordeste R \$ 114,00 (DP95\% 176,64); ao passo que a região Sul aplicou $\mathrm{R} \$$ 162,43 (DP95\% 147,74).

A tabela 3 mostra os dados dos municípios brasileiros que informaram um gasto total com atenção primária inferior ao valor transferido pela União quando o gasto é avaliado por meio do bloco de financiamento da Atenção Básica do Siops, excluídos os municípios que não informaram seus gastos. De modo geral, observa-se a tendência de diminuição da frequência de inexecução das transferências federais ao longo do período analisado - de 18,23\% em 2008 para 13,28\% em 2010, na média nacional. No entanto, ressalta-se que a frequência de inexecução ainda foi relativamente alta (10,75\% na média nacional) no período 2008-2010. Essa tendência também é verificada nos grupos homogêneos e nas regiões.

Tabela 3. Percentual de municípios que não executaram o valor transferido pela União, por grupo homogêneo e por região brasileira, Brasil, 2008 a 2010

\begin{tabular}{|c|c|c|c|c|}
\hline & 2008 & 2009 & 2010 & 2008-2010 \\
\hline & (a) & (a) & (a) & (a) \\
\hline & \% mun. não exec. & \% mun. não exec. & \% mun. não exec. & \% mun. não exec. \\
\hline \multicolumn{5}{|c|}{ Média Nacional } \\
\hline Brasil & 18,23 & 13,79 & 13,28 & 10,75 \\
\hline \multicolumn{5}{|c|}{ Grupo Homogêneo } \\
\hline 1 & 37,50 & 45,83 & 25,00 & 25 \\
\hline 2 & 29,27 & 26,83 & 17,07 & 19,51 \\
\hline 3 & 19,34 & 16,79 & 12,23 & 12,04 \\
\hline 4 & 29,51 & 14,75 & 14,14 & 15,57 \\
\hline 5 & 15,34 & 11,7 & 10,77 & 9,08 \\
\hline 6 & 16,69 & 16,13 & 15,46 & 9,83 \\
\hline \multicolumn{5}{|c|}{ Região Brasileira } \\
\hline $\mathbf{N}$ & 20,53 & 18,93 & 19,2 & 17,87 \\
\hline NE & 23,24 & 16,85 & 15,94 & 12,22 \\
\hline $\mathrm{CO}$ & 11,14 & 7,67 & 8,91 & 7,92 \\
\hline SE & 13,69 & 13,69 & 12,12 & 7,95 \\
\hline s & 19,11 & 14,13 & 10,53 & 10,71 \\
\hline
\end{tabular}

Fonte: Elaboração própria.

Nota: (a) porcentagem dos municípios que não executaram o valor transferido pela União. 
Especificamente aos grupos homogêneos, a inexecução das transferências da União pelos municípios para a atenção primária no período 2008-2010 foi maior no grupo homogêneo $1(25,0 \%)$ e menor no grupo homogêneo 5 (9,08\%). Em relação às regiões brasileiras, no mesmo período, o Norte e o Nordeste foram as regiões que menos executaram o valor transferido pela União direcionado à atenção primária $(17,87 \%$ e 12,22\%, respectivamente), enquanto o Centro-Oeste apresentou um menor percentual de municípios com inexecução dos repasses federais (7,92\%) (tabela 3).
A tabela 4 apresenta a distribuição dos municípios brasileiros nas quatro categorias (AA, $\mathrm{AB}, \mathrm{BA}$ e $\mathrm{BB}$ ), identificando os municípios eficientes e ineficientes com respeito à atenção primária. Nacionalmente, a maior parte dos municípios $(44,7 \%)$ encontra-se na categoria $\mathrm{AB}$, considerada como a mais eficiente, por representar a melhor relação gasto/desempenho. O percentual de municípios ineficientes (categoria BA) é de $8,72 \%$. Foi possível observar também que $78,11 \%$ dos municípios brasileiros apresentam alto IDSUS-AP. Entre os municípios com baixo IDSUS-AP, 80,36\% apresentam também baixo gasto.

Tabela 4. Resultados da análise de eficiência dos municípios brasileiros segundo categorias $A A, A B, B A$ e $B B$, por grupos homogêneos e regiões brasileiras, Brasil, 2008 a 2010

\begin{tabular}{|c|c|c|c|c|}
\hline & AA $\%$ & $A B \%$ & BA $\%$ & BB $\%$ \\
\hline & $\begin{array}{l}\text { Alto IDSUS-AP e alto } \\
\text { gasto }\end{array}$ & $\begin{array}{l}\text { Alto IDSUS-AP e baixo } \\
\text { gasto }\end{array}$ & $\begin{array}{l}\text { Baixo IDSUS-AP e alto } \\
\text { gasto }\end{array}$ & $\begin{array}{c}\text { Baixo IDSUS-AP e baixo } \\
\text { gasto }\end{array}$ \\
\hline \multicolumn{5}{|c|}{ Média Nacional } \\
\hline Brasil & 26,47 & 35,81 & 9,49 & 28,23 \\
\hline \multicolumn{5}{|c|}{ Grupo Homogêneo } \\
\hline 1 & 12,45 & 8,33 & 8,33 & 70,83 \\
\hline 2 & 6,09 & 12,19 & 8,53 & 73,17 \\
\hline 3 & 17,15 & 19,52 & 15,51 & 47,81 \\
\hline 4 & 9,83 & 36,06 & 3,89 & 50,2 \\
\hline 5 & 42,24 & 33,21 & 12,24 & 12,29 \\
\hline 6 & 32,48 & 43,12 & 6,97 & 17,42 \\
\hline \multicolumn{5}{|c|}{ Regiões Brasileiras } \\
\hline $\mathbf{N}$ & 18,4 & 24 & 9,06 & 48,26 \\
\hline NE & 21,78 & 47,66 & 5,61 & 24,85 \\
\hline $\mathrm{CO}$ & 38,61 & 26,48 & 15,09 & 19,55 \\
\hline SE & 40,78 & 25,67 & 12,05 & 21,4 \\
\hline S & 43,21 & 29,17 & 9,97 & 17,54 \\
\hline
\end{tabular}

Fonte: Elaboração própria.

Notas: $A A$, municípios com alto desempenho e alto gasto total médio per capita; $A B$, municípios com alto desempenho e baixo gasto total médio per capita; BA, municípios com baixo desempenho e alto gasto total médio per capita; BB, municípios com baixo desempenho e baixo gasto total médio per capita.

Nos grupos homogêneos 1, 2, 3 e 4 prevaleceram os municípios da categoria $\mathrm{BB}$, chegando a mais de $70 \%$ nos grupos 1 e 2 . $\mathrm{Na}$ categoria dos mais eficientes $(\mathrm{AB})$, destaca-se o grupo 6, que obteve o maior percentual de municípios $(43,12 \%)$. Em termos regionais, por sua vez, o Norte e o Nordeste obtiveram quase metade de seus municípios em uma categoria, sendo o Norte na BB $(48,26 \%)$ e o Nordeste na AB (47,66\%). Cabe destacar 
que o Nordeste também apresentou o menor percentual de municípios na categoria BA, com $5,61 \%$ do total desta região.

\section{Discussão}

Os resultados deste estudo apontam as seguintes observações: i) a dificuldade de estruturação da atenção primária nas grandes cidades (grupos homogêneos 1 e 2) e no Norte; ii) as transferências federais favoreceram os municípios com maiores necessidades, visando à equidade, em termos tanto dos grupos homogêneos quanto das regiões; iii) a existência de inexecução orçamentária em alguns municípios brasileiros referente à atenção primária, principalmente no grupo homogêneo 1 e nas regiões Norte e Nordeste, quando avaliados os gastos informados pelos municípios através do bloco de financiamento da Atenção Básica no Siops; iv) a grande contribuição em termos de eficiência do grupo homogêneo 6 e do Nordeste na relação gasto/desempenho da atenção primária.

A cobertura populacional da atenção primária no grupo homogêneo 1 e 2 ainda é muito baixa. Essa é uma situação antiga, dado que desde 2002 observa-se que a ESF tem tido dificuldade de ser introduzida de forma ampla e substitutiva ao modelo assistencial tradicional nos grandes centros urbanos, com sistemas de saúde complexos. Nessas cidades, a Estratégia ainda é focalizada apenas em grupos populacionais de maior risco social e por meio apenas da expansão de cuidados mínimos (CAETANO; DAIN, 2002). Alguns desafios decorrem das desigualdades sociais e de acesso aos serviços de saúde e das diferentes combinações do mix públicoprivado nesses municípios, que acabam por concorrer para a consolidação do modelo de atenção primária preventiva (VIANA ET AL., 2008).

$\mathrm{O}$ único indicador que foi melhor no grupo homogêneo 1 do que nos demais é o de internação sensível à atenção primária, considerado um indicador utilizado para avaliar os serviços de saúde e a capacidade de resolução desse nível de cuidado; assim, o acesso à atenção primária de qualidade razoável deveria reduzir esse indicador (ANSARI; LADITKA; LADITKA, 2006). Porém, alguns autores argumentam que essas internações podem estar mais relacionadas a variáveis socioeconômicas do que à efetividade da atenção primária, particularmente devido aos determinantes sociais do processo saúde-doença (REHEM, 2011).

As transferências federais para a atenção primária demonstraram ser realizadas de acordo com a equidade. Os grupos homogêneos 6, 5 e 4 e as regiões Norte e Nordeste receberam maior transferência per capita. Tanto a equidade quanto a ampliação do valor per capita transferido pela União são reforçados a partir de 2003 (CASTRO; MACHADO, 2010). Nesse ano, o Ministério da Saúde estabeleceu uma estratégia de correção progressiva, aumentando o PAB fixo, valor transferido per capita, sem necessidade de pactuações, a exemplo do que ocorre no $\mathrm{PAB}$ variável. Além disso, com o objetivo de atingir um financiamento mais equitativo, a Portaria do Ministério da Saúde GM/MS n ${ }^{\circ}$ $1.434 / 2004$, pela primeira vez, empregou indicadores de condições sociais como critério para diferenciar os repasses federais aos municípios (SOLLA ET AL., 2007).

Apesar da transferência federal mais equitativa para a atenção primária nas regiões Norte e Nordeste, a execução orçamentária não é adequada: essas são as regiões com maior percentual de inexecução do valor transferido pela União para os municípios por meio do bloco de financiamento da Atenção Básica. Tal execução ocorre também no grupo homogêneo 1. Uma possível razão para essa baixa execução orçamentária é a disponibilidade insuficiente de profissionais de saúde, especialmente médicos para atuarem nessas regiões, o que dificulta a aplicação dos recursos (MAFRA, 2011). Outro elemento que os municípios alegam para justificar a baixa execução orçamentária da atenção primária é a transferência de 
valores excessivamente 'carimbados' pela União, isto é, de destinação muito fechada e nem sempre de acordo com a necessidade da realidade local (ASSIS ET AL., 2007).

Existe ainda a possibilidade que, como os dados de execução orçamentária são declarados pelos municípios, a informação esteja incorreta; isto é, apesar de os municípios receberem o recurso federal por meio do bloco de financiamento da Atenção Básica, podem estar informando o gasto em outro bloco. Ainda assim, sendo o Siops único no seu propósito, resta-nos avaliar o financiamento e a execução orçamentária com APS por meio dos dados ali contidos e trazer ao conhecimento esse possível problema, que exige uma melhor adequação da contabilidade dos municípios. Especialmente porque o Art $6^{\circ}$. da Portaria GM/MS n. 204/2007determina que "os recursos referentes a cada bloco de financiamento devem ser aplicados nas ações e serviços de saúde relacionados ao próprio bloco" (BRASIL, 2007), não é possível o uso do recurso federal em bloco distinto do inicialmente determinado.

A contrapartida municipal/estadual é outro elemento importante do financiamento da atenção primária. Neste estudo, verificaram-se grandes diferenças na proporção entre o gasto total médio e o valor federal transferido para a atenção primária entre 2008 e 2010: a região Norte aplicou 1,45 vez o valor transferido, enquanto a região Sul aplicou 3,34 vezes. A baixa aplicação própria das regiões Norte e Nordeste era esperada, por serem regiões com menor arrecadação tributária, tendo, portanto, pouca capacidade de aplicação de recursos próprios (PIOLA ET AL., 2009).

Nos grandes centros urbanos que compõem os grupos 1 e 2 , existe uma preponderância de assistência de média e alta complexidade, com precária oferta de atenção primária; nesses municípios, o orçamento e a força de trabalho são voltados para os níveis secundários e terciários, não havendo prioridade ao primário (ASSIS ET AL., 2007). Isso pode ocorrer pela dificuldade em conciliar a organização desses níveis de atenção - com maior densidade tecnológica, o que demanda muitos recursos financeiros e humanos - com a estruturação da atenção primária. Além dessas dificuldades, existe ainda uma cobrança social que constantemente demanda mais recursos para a média e alta complexidade, direcionando a escolha política dos gestores no investimento dos recursos, que acaba por precarizar a atenção primária.

A maior parte dos municípios da região Nordeste e do grupo homogêneo 6 (onde estão $71,32 \%$ dos municípios nordestinos) encontra-se na categoria $\mathrm{AB}$. A eficiência (relação menor gasto com maior desempenho) nordestina na atenção primária também foi observada em outro estudo realizado com dados de 2008, que utilizou indicadores do Pacto pela Saúde e o gasto realizado pelo município, por meio da metodologia $D E A$, em 3.892 municípios brasileiros; ademais, as capitais foram consideradas ineficientes (DIAS, 2010). A eficiência do Norte também foi observada naquele estudo, sendo um ponto de divergência com nossos resultados.

Uma possível explicação para o Nordeste ser mais eficiente pode ser a comparação da alta adesão à ESF às outras regiões. Na média do período 2007-2010, a cobertura populacional por agentes comunitários de saúde e por equipes de saúde da família foi de $85,7 \%$ e $69,8 \%$, respectivamente, no Nordeste. Nas demais regiões, somente o Norte se aproximou da cobertura por agentes comunitários $(80,8 \%)$, estando as demais em torno de $\mathbf{5 5 \%}$, e, na cobertura por equipe saúde da família, a média das demais regiões é bem inferior ao Nordeste, em torno de $46 \%$ (BRASIL, 2015). Já se havia observado que o modelo de saúde da família está associado a melhor desempenho quando comparado com o modelo de atenção primária tradicional (FACCHINI ET AL., 2006).

Algumas limitações relacionadas aos resultados deste estudo devem ser ressaltadas. A primeira está relacionada à opção de utilizar a média dos valores declarados pelos municípios de 2008 a 2010, tendo o ano de 
2007 sido excluído da análise porque 79,3\% dos municípios não declaram o valor despendido na atenção primária, o que enviesaria substancialmente a média amostral dessa variável; inicialmente, o ano de 2007 seria utilizado para fazer correspondência temporal com o IDSUS-AP. A segunda advém da forma como esse índice foi calculado - a partir de coleta de indicadores ao longo de quatro anos (2007-2010) de forma pontual -, o que impediu a obtenção de uma tendência de evolução dos indicadores ao longo dos quatro anos. No entanto, esse é o único índice de desempenho da atenção primária disponível no Brasil que se aplica em nível nacional e para todos os municípios brasileiros.

\section{Conclusão}

Este estudo avaliou a relação entre o gasto total médio per capita e o desempenho, ambos relacionados com a atenção primária, nos municípios brasileiros no período 20072010, com vistas a identificar a eficiência e possíveis disparidades. O Nordeste foi a região que obteve o maior número de municípios considerados eficientes na atenção primária (47,66\%), isto é, apresentou a melhor relação de menor gasto com maior desempenho. Resultado semelhante foi encontrado para o grupo de municípios com os piores indicadores socioeconômicos e de estrutura de saúde (grupo homogêneo 6), para o qual $43,12 \%$ dos municípios foram considerados eficientes. Em termos de disparidade, o Norte apresentou a maior proporção de municípios $(48,26 \%)$ com baixo desempenho e baixo gasto total médio per capita na atenção primária, bem como baixo desempenho e gastos, além de melhores indicadores socioeconômicos e de estrutura de saúde (grupo homogêneo 1), com 70,83\%. Também foi encontrada inexecução orçamentária entre 2008 e 2010 (10,75\%), mostrando que alguns municípios encontraram dificuldades em executar o valor transferido pela União para a atenção primária ou não informaram de maneira correta seus gastos com APS por meio do bloco de financiamento da Atenção Básica, sendo que se destacaram os municípios da região Norte e Nordeste e aqueles com melhores indicadores socioeconômicos e de estrutura de saúde (grupos homogêneos 1). Essas informações são importantes para orientar o planejamento das políticas públicas de saúde, especialmente com relação ao seu financiamento e à promoção da redução das desigualdades regionais com o objetivo de aprimorar de forma equitativa e eficiente a atenção primária no Brasil. Podem, ainda, ser importantes para orientar a adequada capacitação dos municípios sobre como informar seus gastos no Siops.

\section{Referências}

ALVES, L. A.; COSTA, L. A. Avaliação da eficiência na Atenção Básica à saúde nos municípios do estado do Espírito Santo. In: ENCONTRO DA ANPAD, 37., 2013. Rio de Janeiro. Anais... Rio de Janeiro, 2013. Disponível em: <http://www.fucape.br/_public/producao_cientifica/2/APB565-\%20Luiz\%20Antonio\%20Alves.pdf>. Acesso em: 7 jan. 2015.
ANSARI, Z.; LADITKA, J. N.; LADITKA, S. B. Access to health care and hospitalization for ambulatory care sensitveconditions. MedCare Res Rev, Thousand Oaks, v. 63, n. 6, p. 719-741, 2006. Disponível em: <http:// www.ncbi.nlm.nih.gov/pubmed/17099123>. Acesso em: 20 dez. 2014. 
ASSIS, M. M. A. et al. Atenção Primária à Saúde e sua articulação com a estratégia saúde da família: construção política, metodológica e prática. Rio de Janeiro: Fiocruz, 2007. Disponível em: <http://www5.ensp.fiocruz.br/biblioteca/dados/txt_228616371.pdf>. Acesso em: 10 jan. 2014.

BRASIL. Ministério da Saúde. Índice de Desempenho do SUS. Brasília, DF: Ministério da Saúde, 2012. Disponível em: <http://idsus.saude.gov.br/>. Acesso em: 10 out. 2014.

Ministério da Saúde. Departamento de Atenção Básica. Histórico de cobertura da saúde da família. Brasília, DF: Ministério da Saúde, 2015. Disponível em: $<$ http://dab.saude.gov.br/portaldab/historico_cobertura_sf.php >. Acesso em: 8 jan. 2015.

Ministério da Saúde. Portaria GM/MS 204. Brasília, DF: Ministério da Saúde, 2007. Disponível em: <http://bvsms.saude.gov.br/bvs/saudelegis/gm/2007/ prt0204_29_01_2007_comp.html>. Acesso em: 10 out. 2014.

Tribunal de Contas da União. Relatório Sistêmico de Fiscalização da Saúde. Brasília, DF, 2012b. Disponível em: <http://portal2.tcu.gov.br/portal/ page/portal/TCU/imprensa/noticias/noticias_arquivos/032.624-2013-1\%20Fisc\%20Saude.pdf >. Acesso em: 10 jan. 2015

BROUSSELLE, A.; LACHAINE, J.; CONTANDRIOPOULOS, A. P. A Avaliação Econômica. In: BROUSSELLE, A. (Org.). Avaliação: conceitos e métodos. Rio de Janeiro: Editora Fiocruz, 2013.

CAETANO, R.; DAIN, S. O Programa Saúde da Família e a reestruturação da Atenção Básica à saúde nos grandes centros urbanos: velhos problemas, novos desafios. Physis, Rio de Janeiro, v. 12, n. 1, p. 11-21, 2002. Disponível em: <http:/www.scielo.br/pdf/physis/ v12nl/a02.pdf $>$. Acesso em: 8 jan. 2015.

CASTRO, A. L. B.; MACHADO, C. V. A política de atenção primária à saúde no Brasil: notas sobre a regulação e o financiamento. Cad. Saúde Pública, Rio de Janeiro, v. 26, n. 4, 2010. Disponível em:
$<$ http://www.scielo.br/scielo.php?pid=S0102$-311 X 2010000400012 \&$ script $=$ sci_arttext $>$. Acesso em: 21 dez. 2014

DIAS, R. H. Eficiência da atenção primária a saúde nos municípios brasileiros. 2010. 42 f. Dissertação (Mestrado em Economia) - Universidade de Brasília, FEAC, Brasília, DF, 2010. Disponível em: <http://repositorio.unb.br/handle/10482/8364>. Acesso em: 13 jan. 2015

DONABEDIAN, A. An Introduction to Quality Assurance in Health Care. Oxford: Oxford University Press, 2003.

FACCHINI, L. A. et al. Desempenho do PSF no Sul e no Nordeste do Brasil: avaliação institucional e epidemiológica da Atenção Básica à Saúde. Ciênc. saúde colet, Rio de Janeiro, v. 11, n. 3, 2006. Disponível em: < http:// www.scielo.br/pdf/csc/vlln3/30982.pdf >. Acesso em: 5 jan. 2015

\section{INSTITUTO BRASILEIRO DE GEOGRAFIA E} ESTATÍSTICA (IBGE). Índice Nacional de Preços ao Consumidor. 2014. Disponível em: <http://www.ibge. gov.br/home/estatistica/indicadores/precos/inpc ipca/defaultinpc.sht>. Acesso em: 4 jan. 2015.

MAFRA, F. O impacto da Atenção Básica em saúde em indicadores de internação hospitalar no Brasil. 2011. 129f. Dissertação (Mestrado Profissional em Gestão de Negócios) - Universidade de Brasília, Brasília, DF, 2011. Disponível em: <http://portal2.tcu.gov.br/portal/pls/ portal/docs/2054336.PDF>. Acesso em: 5 jan. 2015.

MENDES, A.; MARQUES, R. M. O financiamento da Atenção Básica e da Estratégia Saúde da Família no Sistema Único de Saúde. Saúde em Debate, Rio de Janeiro, v. 38, n. 103, p. 900-916, out./dez., 2014.

PIOLA, S. F. et al. Saúde no Brasil: algumas considerações sobre o sistema único de saúde (SUS). Brasília, DF: Cepal, 2009. Disponível em: <http://www.cepal. org/brasil/publicaciones/sinsigla/xml/4/35734/ LCBRSR200SaudenoBrasil.pdf $>$. Acesso em: 10 jan. 2015 . 
REHEM, T. C. M. S. B. Internações sensíveis à atenção primária: limites e possibilidades da lista Brasileira de diagnósticos. 2011. 308 f. Tese. (Doutorado em Enfermagem) - Universidade de São Paulo, São Paulo, 2011. Disponível em: <http://www.teses.usp.br/teses/ disponiveis/83/83131/tde-27012012-124850/pt-br.php>. Acesso em: 20 dez. 2014.

RODRIGUES, L. B. B. et al. A atenção primária à saúde na coordenação das redes de atenção: uma revisão integrativa. Ciênc. saúde colet., Rio de Janeiro, v. 19, n. 2, p. 343-352, 2014.

\section{SANTOS, L. M., GONÇALVES, M. A., FIGUEIREDO,}

A. C. Avaliação de desempenho da alocação de recursos públicos na Atenção Básica da saúde: o caso da região sudeste do Brasil. In: ENCONTRO DA ANPAD, 2013.

Rio de Janeiro. Anais... Rio de Janeiro, 2013. Disponível em: <http://www.anpad.org.br/admin/pdf/2013_

EnANPAD_APB842.pdf>. Acesso em: 7 jan. 2015.

SOLLA, J. J. S. P. et al. Mudanças recentes no financiamento federal do Sistema Único de Saúde: Atenção Básica à saúde. Rev. Bras. Saude Matern. Infant, Recife, v. 7, n. 4, out./dez., 2007. Disponível em: <http:// www.scielo.br/scielo.php?script=sci_arttext\&pid $=$ S1519-38292007000400018>. Acesso em: 21 dez. 2014.
VARELA, P. S., MARTINS, G. A., FÁVERO, L. P. L. Desempenho dos municípios paulistas: uma avaliação de eficiência da Atenção Básica à saúde. Rev. Adm., São Paulo, v. 47, n. 4, 2012. Disponível em: <http:// www.scielo.br/scielo.php?script=sci_arttext\&pid =S0080-21072012000400009>. Acesso em: 7 jan. 2015 .

VIACAVA, F. et al. Avaliação de Desempenho de Sistemas de Saúde: um modelo de análise. Ciênc. saúde colet., Rio de Janeiro, v. 17, n. 4, 2012. Disponível em: <http://www.scielo.br/scielo.php?pid=S1413$-81232012000400014 \&$ script $=$ sci_arttext $>$. Acesso em: 4 jan. 2015.

VIANA, A. L. A. et.al. Atenção básica e dinâmica urbana nos grandes municípios paulistas, Brasil. Cad. Saúde Pública, Rio de Janeiro, v. 24, n. 1, 2008. Disponível em: <http://www.scielo.br/scielo.php?script=sci_artte $\mathrm{xt} \& \mathrm{pid}=\mathrm{S} 0102311 \mathrm{X} 2008001300013 \& \operatorname{lng}=\mathrm{en} \& \mathrm{nrm}=\mathrm{iso}>$. Acesso em: 9 jan. 2015.

Recebido para publicação em junho de 2015

Versão final em agosto de 2015

Conflito de interesses: inexistente

Suporte financeiro: não houve 